\title{
Universality at work - the local sine-Gordon model, lattice fermions, and quantum circuits
}

\author{
Anne Anthore ${ }^{1,2}$, Dante M. Kennes ${ }^{3}$, Edouard Boulat ${ }^{4}$, Sabine Andergassen ${ }^{5}$, \\ Frédéric Pierre ${ }^{1}$, and Volker Meden ${ }^{3, a}$ \\ ${ }^{1}$ Centre de Nanosciences et de Nanotechnologies (C2N), CNRS, Univ Paris Sud, \\ Université Paris-Saclay, 91120 Palaiseau, France \\ ${ }^{2}$ Université de Paris, Univ Paris Diderot, 75013 Paris, France \\ ${ }^{3}$ Institut für Theorie der Statistischen Physik, RWTH Aachen University and \\ JARA-Fundamentals of Future Information Technology, 52056 Aachen, Germany \\ ${ }^{4}$ Université de Paris, Laboratoire Matériaux et Phénomènes Quantiques (MPQ), \\ Univ Paris Diderot, CNRS, 75013 Paris, France \\ ${ }^{5}$ Institut für Theoretische Physik and Center for Quantum Science, Universität \\ Tübingen, 72076 Tübingen, Germany
}

Received 18 June 2019 / Received in final form 12 August 2019 Published online 14 February 2020

\begin{abstract}
We review the intriguing many-body physics resulting out of the interplay of a single, local impurity and the two-particle interaction in a one-dimensional Fermi system. Even if the underlying homogeneous correlated system is taken to be metallic, this interplay leads to an emergent quantum phase transition between metallic and insulating states. We show that the zero temperature critical point and the universal low-energy physics associated to it, is realized in two different models, the field theoretical local sine-Gordon model and spinless fermions on a lattice with nearest-neighbor hopping and two-particle interaction, as well as in an experimental setup consisting of a highly tunable quantum circuit. Despite the different high-energy physics of the three systems the universal low-energy scaling curves of the conductance as a function of temperature agree up to a very high precision without any free parameter. Overall this provides a convincing example of how emergent universality in complex systems originating from a common underlying quantum critical point establishes a bridge between different fields of physics. In our case between field theory, quantum many-body theory of correlated Fermi systems, and experimental circuit quantum electrodynamics.
\end{abstract}

\section{An impurity in a one-dimensional, correlated Fermi system}

\subsection{Linear response theory}

Linear response theory provides a framework to study the effect of a weak local impurity in a nonrelativistic many-body Fermi system. Let us assume that we are

a e-mail: meden@physik.rwth-aachen.de 
interested in the change of the particle density in response to the introduction of an impurity potential into a translational invariant system. In this case we need to consider the density-density response function $\chi(q, \omega)$ of the homogeneous system. In one spatial dimension (1d) and in the absence of two-particle interaction this Lindhard function at temperature $T=0$ and in the static limit, i.e. at energy $\omega=0$ (measured relative to the chemical potential), shows a logarithmic divergence if the momentum $q$ approaches twice the Fermi momentum $k_{\mathrm{F}}$ [1]. This divergence results from the restricted phase space available in $1 \mathrm{~d}$ and is thus not specific to any particular model such as, e.g., the 1d Fermi gas. The divergence indicates that any impurity with a nonvanishing backscattering $2 k_{\mathrm{F}}$ component strongly affects the density. Taking into account that in $1 \mathrm{~d}$ particles cannot bypass the impurity this insight might be considered as intuitive.

In 1974 the density-density response function of the spinless Fermi gas complemented by a two-particle interaction was computed $[2,3]$. It was shown that the logarithmic singularity of the noninteracting case turns into a power law with an exponent which can be expressed in terms of an interaction dependent parameter $K$, the so-called Tomonaga-Luttinger liquid parameter (see below),

$$
\chi\left(q \approx 2 k_{\mathrm{F}}, 0\right) \sim\left|q-2 k_{\mathrm{F}}\right|^{2(K-1)} .
$$

For repulsive two-particle interactions $0<K<1$ holds while $K>1$ for attractive ones. The power-law divergence in the repulsive case shows that the interacting homogeneous system is perturbed even more strongly by a single impurity than the noninteracting one. It, in fact, indicates that linear response theory breaks down. The response of the homogeneous system to the impurity potential is large even if its amplitude at momentum transfer $2 k_{\mathrm{F}}$ is arbitrarily small. Thus more elaborate methods than linear response theory are required to study the effect of impurity backscattering in 1d correlated Fermi systems. Before returning to this issue in Section 1.3 we will next discuss the physics of homogeneous interacting 1d Fermi systems from a more general perspective. This turns out to be a necessary first step.

\subsection{Homogeneous Tomonaga-Luttinger liquids}

Power-law scaling of correlation functions as in equation (1) is characteristic for translationally invariant, metallic, and interacting 1d Fermi systems. Employing renormalization group ( $\mathrm{RG}$ ) arguments, one can show that the Tomonaga-Luttinger model is the low-energy fixed point of a large class of models in which the interaction does not lead to the opening of a gap $[4,5]$. This class includes the continuum electron gas with two-particle interaction but also lattice models, such as, e.g., spinless fermions with nearest-neighbor hopping and nearest-neighbor interaction. The Tomonaga-Luttinger model plays the same role in 1d as the noninteracting Fermi gas does for (interacting) higher dimensional metallic systems. The noninteracting Fermi gas is the fixed-point model of systems falling into the Fermi liquid universality class.

To understand the universal low-energy physics of Tomonaga-Luttinger liquids, in a first step, one can thus study the Tomonaga-Luttinger model. It has two branches of fermions (right- and left-moving ones) with linear dispersion and two-particle scattering which is restricted to small momentum transfer $|q| \ll k_{\mathrm{F}}$. The elementary low-energy excitations of this model are not given by fermionic quasi-particles, as in Fermi liquids, but are of collective bosonic nature. Using bosonization [5-7] thermodynamic observables and all correlation functions of interest can be computed exactly in the low-energy scaling limit. For the spinless case, on which we focus, the low-energy physics is characterized by only two parameters, $K$ and the renormalized velocity $v$. The parameters appearing in the Hamiltonian of the Tomonaga-Luttinger 
model enter the exponents of the characteristic power laws of correlation functions only via $K$. The exponent of each correlation function is given by a unique function of $K$. For an example, see equation (1).

To determine the low-energy physics of a given microscopic model from the Tomonaga-Luttinger liquid universality class one can, in a second step, proceed as follows. By computing two thermodynamic observables, e.g., the compressibility and the specific heat, $K$ and $v$ can be determined in terms of the microscopic parameters. In particular, these are the amplitude and range of the two-particle interaction, the parameters of the single-particle dispersion (such as the hopping amplitude in a tightbinding model), and the band filling. $K$ and $v$ can then be plugged into the expressions of the correlation functions and observables of the Tomonaga-Luttinger model. The challenging explicit computation of correlation functions for an interacting microscopic model of interest can be avoided this way. Thermodynamic observables are easier to access either by analytical means such as perturbation theory and the Bethe ansatz, or by numerical approaches $[5,7]$.

For the above mentioned lattice model of spinless fermions, which will be one of the models considered here, $K$ and $v$ as functions of the nearest-neighbor interaction $U$, the nearest-neighbor hopping $t$, and the filling $\nu$ can be computed exactly (see below) employing the Bethe ansatz expression for the ground state energy [8]. At half filling $\nu=1 / 2$ the corresponding set of integral equations can be solved analytically while away from half filling a numerical solution up to very high precision is possible. For $\nu \neq 1 / 2$ the model is from the Tomonaga-Luttinger liquid universality class for all $U / t>-2$, while for $\nu=1 / 2$ this low-energy physics is only found for $-2<U / t<2$. In Section 3 we will return to this model.

For many years emergent universal Tomonaga-Luttinger liquid physics of $1 \mathrm{~d}$ systems was considered to be an appealing theoretical concept, which, however, was far from being realizable in real-world experiments. Only at the beginning of the 1990s material science and nanostructuring techniques reached a level, such that an experimental realization appeared to be within reach. Promising systems to observe the typical Tomonaga-Luttinger liquid power-law behavior of the spectral and transport properties are highly anisotropic quasi 1d crystals, semi-conductor-based quasi 1d heterostructures (cleaved-edge overgrowth), self-organized atom chains on surfaces, and unidirectional long molecules, such as, e.g., metallic carbon nanotubes. In fact, examples from all these classes were investigated concerning their TomonagaLuttinger liquid properties (for reviews, see Ref. [9-11]). Although many of the measurements are consistent with Tomonaga-Luttinger liquid behavior, convincing examples of power-law scaling in an energy variable, such as the temperature $T$ or the frequency $\omega$, are rare. In most experiments the energy regime over which results consistent with power-law behavior can be observed is small, typically less than one order of magnitude. At the lower end the power laws are cut off by a finite energy resolution or effects beyond the Tomonaga-Luttinger liquid theory such as coupling of the $1 \mathrm{~d}$ chains. At the higher end details of the experimental system, e.g. the band structure, cut off the universal low-energy physics. Having only a small window of energies available and taking into account experimental noise, it is hardly possible to distinguish power-law behavior from other functional forms. In addition, in most systems, it is impossible to control any other parameter besides the energy variable in which power-law scaling is investigated, e.g. the strength of the two-particle interaction. It is thus impossible to show consistency of the experimentally extracted exponents with the predictions of Tomonaga-Luttinger liquid theory. In short, the systems lack control and tunability. This calls for further attempts to realize Tomonaga-Luttinger liquid physics in experiments. One way is to use other systems, e.g. quantum circuits, to emulate Tomonaga-Luttinger liquid behavior (see, e.g. Ref. [12]). Here we will follow this route but consider inhomogeneous Tomonaga-Luttinger liquids instead of translational invariant ones. 


\subsection{Impurity backscattering}

In a first step towards a comprehensive understanding of the effect of a localized impurity in a Tomonaga-Luttinger liquid beyond linear response theory (see Sect. 1.1), in 1982 Apel and Rice studied a system in which the Tomonaga-Luttinger model is complemented by a pure single-particle backscattering term [13]. Rewritten in terms of the bosonic fields of the bosonization approach it becomes obvious that at low energies this model is equal to the local sine-Gordon model studied in quantum field theory [5-7]. Apel and Rice used a RG-related scaling theory. Their results indicate that for a repulsive two-particle interaction with $0<K<1$ even a weak impurity drives the system from being a metal with finite (dc) conductance $G$ at vanishing $T$ into an insulating phase with $G(T=0)=0$. For attractive interactions the system, in contrast, remains metallic.

In 1992 this picture was confirmed by Kane and Fisher [14]. They studied the local sine-Gordon model for arbitrary $K$ using a $R G$ approach perturbative in the backscattering amplitude. This calculation was complemented by a perturbative RG for the (dual, see Ref. [14]) problem of a weak link connecting two semi-infinite wires each modeled by the Tomonaga-Luttinger Hamiltonian. It turned out that the perfect chain fixed point with vanishing impurity backward scattering is stable for attractive two-particle interactions with $K>1$, but unstable for repulsive ones with $0<K<1$, while the cut chain fixed point, with $G=0$, is stable in the repulsive case and unstable for attractive interactions. For $K=1 / 2$ it was possible to show that both fixed points are directly linked, i.e. not separated by any intermediate fixed point. The consequences of this behavior for observables is best illustrated considering the temperature dependence of the linear conductance. We will discuss this in the next subsection. We note that in their analysis Kane and Fisher were able to resort to results obtained for Hamiltonians studied earlier in the field of dissipative quantum systems $[15,16]$.

The absence of any intermediate fixed point in the local sine-Gordon model with $K=1 / n, n \in \mathbb{N}$, was confirmed numerically by quantum Monte Carlo approaches $[17,18]$ as well as analytically by the Bethe ansatz solution [19]. This let to the general expectation that the same will hold for the local sine-Gordon model with arbitrary $K$. Very recently a modified Bethe ansatz was used to solve the local sine-Gordon model first for $K=2 / 3$ [20] and later for all rational $K<1$ [21]. We will elaborate on these solutions in Section 2.

The just described physics of the local sine-Gordon model is often phrased as follows. For repulsive interactions and on small energy scales even a weak impurity grows and effectively cuts the chain into two parts. The opposite holds for attractive interactions. Even starting with only a weak link connecting two semi-infinite chains (i.e. a strong impurity) the system is "healed", that is, the impurity vanishes.

In the early to mid 1990s two important steps were taken, to show that this intriguing many-particle physics, resulting out of the interplay of single-particle backscattering and the two-particle interaction, is also realized in other models for a single impurity in a 1d correlated fermion system than the specific local sine-Gordon model. Numerical results for the above mentioned lattice model of spinless fermions (more precisely, for the equivalent XXZ-Heisenberg model) at small system sizes turned out to be consistent with the above RG flow in the two limits of a weak impurity and a weak link [22]. In addition, a fermionic RG applicable in the limit $K \rightarrow 1$ was set up for the $1 \mathrm{~d}$ continuum electron gas with an impurity and showed the RG flow connecting the two fixed points [23]. However, it took another ten years to develop an approximate $\mathrm{RG}$ method which is capable to capture the full crossover from the perfect to the cut chain fixed point (or vice versa) for a lattice model [24]. In Section 3 we will describe this approach and present results for $G(T)$ obtained this way. 
Based on these insights one can now be certain that the local sine-Gordon model is the effective low-energy model of a large class of impurity Hamiltonians with the bulk part falling into the Tomonaga-Luttinger liquid universality class. To avoid any confusion we emphasize that in microscopic models one has to distinguish two RG flows. The one of the bulk part of a given model towards the Tomonaga-Luttinger model and the flow of the single-particle (impurity) backscattering amplitude.

The above described transition from metallic $G(T=0)>0$ to insulating $G(T=$ $0)=0$ behavior can also be understood within the framework of quantum critical behavior [25]. The Tomonaga-Luttinger liquid parameter $K$ can be used to tune the inhomogeneous system through the $T=0$ quantum phase transition from a metal $K>1$ to an insulator $0<K<1$. The temperature dependence of the conductance, to be discussed in the next section, reflects the scaling away from criticality which is dominated by the quantum critical point. This quantum critical perspective on the problem of a single impurity in a Tomonaga-Luttinger liquid helps to understand the universality across models and experimental systems; see below.

\subsection{The linear conductance and the universal $\beta$-function}

Within the local sine-Gordon model employing the RG approach perturbative in the weak impurity [14] it is straightforward to show that the conductance close to the perfect chain fixed point depends on temperature as

$$
G_{0}-G(T) \sim V_{\mathrm{b}}^{2} T^{2(K-1)}
$$

with $G_{0}$ being the $T=0$ conductance in the absence of the impurity (see below) and $V_{\mathrm{b}}$ the bare (as opposed to renormalized) amplitude of the impurity backscattering. For $K>1$ the exponent is positive and the finite temperature correction vanishes. This indicates the stability of the perfect chain fixed point for attractive interactions. For $0<K<1$, the correction grows. One says that the backscattering renormalizes towards strong coupling. As the calculation leading to equation (2) is controlled for small renormalized backscattering it holds as long as the right hand side remains small. Assuming a fixed $V_{\mathrm{b}}$ this is only the case for not too small temperatures.

A similar analysis close to the cut chain fixed point gives

$$
G(T) \sim t_{\mathrm{wl}}^{2} T^{2(1 / K-1)}
$$

where $t_{\mathrm{wl}}$ is a measure for the bare hopping between the two semi-infinite chains. For $0<K<1$ the conductance vanishes for $T \rightarrow 0$; the cut chain fixed point is stable and the system is an insulator. For $K>1$ the finite $T$ correction to the cut chain conductance $G=0$ grows for decreasing $T$. As the result equation (3) was derived in the weak link limit the right hand side has to stay small and the temperature cannot be taken too small. In other words, the cut chain fixed point is unstable.

As no intermediate fixed point interrupts the RG flow from the perfect to the cut chain fixed point (or vice versa), the limiting behavior of equations (2) and (3) are connected by a unique, $K$-dependent conductance function. More generally, such universal scaling behavior as a function of temperature is expected in the vicinity of continuous quantum phase transitions, when the system is slightly detuned from the quantum critical point [25]. The theory of quantum critical phenomena predicts that all microscopic parameters [e.g. $V_{\mathrm{b}}$ of equation (2) and $t_{\mathrm{wl}}$ of equation (3)] can be encapsulated into a rescaling temperature $T_{0}$, such that the conductance is given by $G_{0} G_{K}\left(T / T_{0}\right)$ with a universal-but $K$-dependent-dimensionless function $G_{K}(x)$ and $x=T / T_{0}$. 
However, the numerical value of $T_{0}$ is a priori unknown, and depends on specific details of the system. In order to perform a direct comparison between different models, in our case the local sine-Gordon model and the lattice model of spinless fermions, as well as a comparison to experiments, it is possible to eliminate $T_{0}$ by considering, instead of $G$, its logarithmic derivative $d G / d \ln T$ that does not depend on the temperature scale. An underlying universal scaling law then implies the existence of a so-called $\beta$-function completely characterizing the conductance renormalization flow through the relation

$$
\beta_{K}=\frac{d g(T)}{d \ln (T)},
$$

with the normalized conductance $g(T)=\frac{G(T)}{G_{0}}$.

In the two limits of equations (2) and (3) we obtain

$$
\beta_{K}=2(1-K)(1-g)
$$

for $1-g \ll 1$ and

$$
\beta_{K}=2(1 / K-1) g
$$

for $g \ll 1$. In these limits $\beta_{K}$ depends on $x=T / T_{0}$ only via $g$. Based on the discussion of Section 1.3 we expect that $\beta_{K}$ is also model independent in the above two limits.

From the exact analytical results for $G(T)$ obtained by Bethe ansatz for the local sine-Gordon model - for details see the next section - it is known that $\beta_{K}$ is a function of $g$ only also for arbitrary $g$, not only in the limits $1-g \ll 1$ and $g \ll 1$. If the idea of quantum critical universality holds and taken that the quantum critical point separating the metal from the insulator is the same in all models of TomonagaLuttinger liquids with impurity backscattering, the same function $\beta_{K}(g)$ should be found in microscopic models for the specific value of $K$ of the underlying homogeneous system.

It is exactly this type of emergent universality which we will demonstrate in the remainder of this minireview. We will explicitly verify that the $\beta$-function of the lattice model of spinless fermions with nearest-neighbor hopping, nearest-neighbor interaction, and an impurity falls on top of the $\beta$-function of the local sine-Gordon model given the same $K$ (but without any free parameter). Furthermore, we will show that the measured conductance of a highly tunable quantum circuit [20], described by a Hamiltonian which can be argued to be equivalent to the local sine-Gordon model at low energy scales, leads to a $\beta$-function also falling on the universal curve. This will complete our "triangle of universality"; see Figure 1 for an illustration. We note that related experimental results were obtained earlier in reference [26], but for a circuit setup which was less tunable and provided access to a single value of $K$ only. Only recently is was possible to close the triangle with the sides being a field theory (the local sine-Gordon model), an interacting, microscopic lattice model, and an experimental quantum circuit for different values of $K$. It exploits the latest technical developments in the Bethe ansatz solution of the local sine-Gordon model [21] and the recently achieved tunability of $K$ in the quantum circuits [20]. In both research fields only now values of $K$ sufficiently close to 1 can be reached, for which controlled results for the $\beta$-function of the interacting fermionic system are available [24].

At the same time, the quantum circuit provides a convincing experimental emulation of (inhomogeneous) Tomonaga-Luttinger liquid physics beyond the limitations discussed in Section 1.2. 


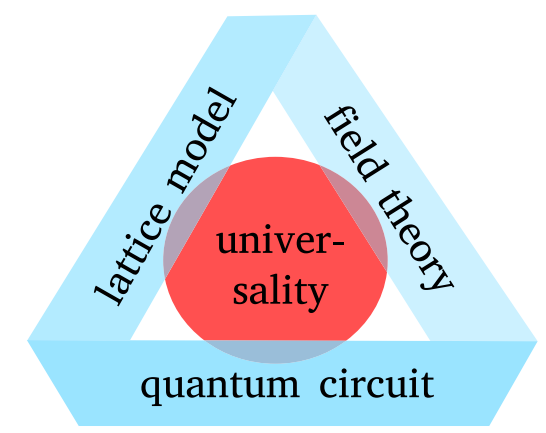

Fig. 1. A sketch of our "triangle of universality". The two models and the experimental system show the same universal low-energy physics resulting out of a common underlying quantum critical point.

We next review how to obtain $G_{K}\left(T / T_{0}\right)$ or, equivalently, $\beta_{K}(g)$ for the local sine-Gordon model.

\section{The local sine-Gordon model}

The local sine-Gordon model is a minimal quantum impurity model, of a free $1 \mathrm{~d}$ massless chiral (say right-moving) boson described by the field $\phi(x)$, with $x \in \mathbb{R}$, which, at the origin, is perturbed by a cosine potential of amplitude $\gamma$

$$
H=H_{0}[\phi]+H_{\mathrm{B}}, \quad H_{0}[\phi]=\int_{-\infty}^{\infty} d x\left(\partial_{x} \phi\right)^{2}, \quad H_{\mathrm{B}}=\gamma \cos [\beta \phi(0)] .
$$

We first discuss how it is related to the problem of a single impurity in a $1 \mathrm{~d}$ fermionic many-body system and, secondly, review its exact solution by means of a Bethe ansatz approach. We consider both, the so-called diagonal case, when $\lambda=\frac{8 \pi}{\beta^{2}}-1 \in \mathbb{N}[19]$, and, the more general off-diagonal case, when $\lambda \in \mathbb{Q}^{+}[21]$. We discuss the current $I$ as a function of $T$ but also as a function of a bias voltage $V$ applied across the impurity (out-of-equilibrium setup). From this the linear conductance $G(T)=\left.\frac{\partial I}{\partial V}\right|_{V=0}$ can be computed.

Employing bosonization the low-energy features of the Tomonaga-Luttinger liquid model are captured by a $1 \mathrm{~d}$ free massless boson theory [5-7], that can be decomposed in terms of chiral right-moving and left-moving modes, with Hamiltonian $H_{0}\left[\phi_{\mathrm{L}}\right]+$ $H_{0}\left[\phi_{\mathrm{R}}\right]$ as in equation (7). Adding an impurity, specifically a local backscattering term, couples $\phi_{\mathrm{L}}$ and $\phi_{\mathrm{R}}$ at $x=0$. The system is described by the local sine-Gordon model equation (7) with $\beta=\sqrt{8 \pi K}$ and $\phi$ essentially being the difference of the bosonic modes on the left and on the right of the impurity [19]. We note in passing that for $K<1 / 4$ the low-energy mapping of the Tomonaga-Luttinger model with local impurity onto the local sine-Gordon model equation (7) is expected to break down [19]. In this case additional allowed and relevant tunneling processes across the impurity corresponding to, e.g., the simultaneous tunneling of two fermions, lead to higher harmonics like $\cos [\sqrt{32 \pi K} \phi(0)]$. Accordingly, universality is lost in this case.

From the scaling dimension $\Delta=K$ of $H_{\mathrm{B}}$ we recover the RG flow discussed in Section 1.4. In the repulsive regime $\Delta<1, H_{\mathrm{B}}$ is a relevant perturbation that cuts the system and at low energy one reaches the insulating fixed-point with vanishing linear conductance. Setting $\gamma=0$ in equation (7) corresponds to the perfect conduction fixed point where the conductance reaches its maximal value $G_{0}$. 
The strong impurity formulation, or cut chain limit, also bears a universal description in terms of a (dual, see Refs. [14,19]) local sine-Gordon model $\widetilde{H}=H_{0}+\widetilde{H}_{\mathrm{B}}$ with $\widetilde{H}_{\mathrm{B}}=\widetilde{\gamma} \cos \left[\sqrt{\frac{8 \pi}{K}} \phi(0)\right]$. Setting $\widetilde{\gamma}=0$ corresponds to the cut chain fixed point, i.e. two semi-infinite Tomonaga-Luttinger models. $\widetilde{H}_{\mathrm{B}}$ describes the tunneling of fermions between the two half chains, and has scaling dimension $\tilde{\Delta}=K^{-1}$. Fermion tunneling is therefore relevant for attractive interactions $K>1$.

A bias voltage $V$ applied across the impurity is described by a term

$$
H_{V}=-e V \sqrt{\frac{K}{2 \pi}} \int_{-\infty}^{\infty} \partial_{x} \phi
$$

which has to be added to the Hamiltonian equation (7). The voltage couples to the charge difference $Q$ between the right and the left of the impurity. The prefactor ensures that $H_{V}=-Q V / 2$. In the absence of the impurity this leads to the conductance

$$
G_{0}=K \frac{e^{2}}{h} .
$$

Note that we add the fundamental constants such as $e, h, \ldots$ to the "theorists units" whenever we judge this to be appropriate.

The potential term in equation (7) generates a typical energy scale, the "impurity temperature" $T_{\mathrm{B}}$, that scales as $T_{\mathrm{B}} \sim W^{\frac{\Delta}{1-\Delta}} \gamma^{\frac{1}{1-\Delta}}$, with the scaling dimension $\Delta=$ $K$. Here $W$ denotes the high-energy cutoff of the model which is left implicit in equation (7) and $T_{\mathrm{B}}$ is the model specific realization of the nonuniversal temperature $T_{0}$ introduced in Section 1.4. Low-energy universality implies that physical quantities $X$ can be expressed as universal functions $f_{\text {univ }}^{(X)}$ of dimensionless arguments $\frac{V}{T_{\mathrm{B}}}, \frac{T}{T_{\mathrm{B}}}, \ldots$, e.g., for the electrical current

$$
I(V, T)=T_{\mathrm{B}} f_{\text {univ }}^{(I)}\left(\frac{V}{T_{\mathrm{B}}}, \frac{T}{T_{\mathrm{B}}}\right),
$$

as long as $V \ll W, T \ll W, \ldots$

The local sine-Gordon model is integrable [27], which allows for a number of exact predictions for the out-of-equilibrium current equation (10) (for arbitrary $\frac{V}{T_{\mathrm{B}}}$ and $\frac{T}{T_{\mathrm{B}}}$ ) for integer values of $\lambda[19]$ and all rational values of $\lambda$ [21]. This provides access to the finite temperature linear conductance. Integrability of the local sine-Gordon model [27-29] and also the bulk sine-Gordon model, implies that both possess a rich mathematical structure, with, amongst other things, an infinity of commuting conserved quantities. Being one of the simplest integrable model, the bulk sine-Gordon model has been studied extensively and the Bethe ansatz approach that will be outlined shortly, builds on developments of formal aspects that have been studied in great detail. A nonexhaustive list of significant contributions include the construction of quasiparticle modes [30,31], and the development of the thermodynamic Bethe ansatz $[32,33]$. In the context of the XXZ spin lattice model, strings and the algebraic Bethe ansatz were introduced [34-37] and adapted to the sine-Gordon model [38-41]. The description of the impurity scattering was included in references [27-29].

Avoiding all tedious technicalities that come along with this rich mathematical structure, in practice one achieves an exact change of the many-body basis from a free chiral boson with bias voltage (states incoming from $x=-\infty$ towards the impurity), to a gas of interacting quasiparticles whose thermodynamics is known 
exactly. Just as in free gases, where many-body states have the structure of Fock states generated by modes $a_{p}^{\dagger}$, creating single-particle plane waves with momentum $p$, the many-body states can be described as collections of quasiparticles created by modes $A_{a}(\theta)$, with quantum number $a$ and momentum $p$ parameterized by a rapidity $\theta=\ln p$. Yet they are interacting quasiparticles, with nontrivial scattering amongst them, which is encoded in the bulk scattering matrix $S$. As a consequence the density $\rho_{a}(\theta)$ describing the thermodynamics of the gas depends in a nonlinear way on the densities of all other particles $\rho_{b}\left(\theta^{\prime}\right)$. The out-of-equilibrium treatment employs integrability and identifies the basis of quasiparticle modes $A_{a}(\theta)$ with the following properties:

1. The many-body states generated by the quasiparticle modes are the many-body states of a chiral right moving boson.

2. The density matrix of the free boson gas at $T$ and $V$ can be represented in terms of the quasiparticle modes.

3. The many-body bulk scattering amongst quasiparticles is factorized.

4. The many-body bulk scattering amongst quasiparticles is diagonal.

5. The many-body impurity scattering is factorized and without particle production, i.e. a single quasiparticle incoming state yields a single quasiparticle outgoing state after impurity scattering.

Finding the basis turns out to be quite different when $\lambda$ is an integer, and when it is a positive rational number.

\section{Diagonal case: $\lambda \in \mathbb{N}$}

The solution to the "integer" local sine-Gordon model [19], with $\lambda \in \mathbb{N}$, involves two quasiparticles, a soliton and an antisoliton $A_{ \pm}(\theta)$, carrying an electric charge $\pm q_{\mathrm{s}}= \pm \sqrt{\frac{8 \pi K}{\beta^{2}}}$ respectively, together with a collection of $n_{\mathrm{b}}=\lambda-1$ neutral bound states $\left(A_{+}, A_{-}\right)$called breathers.

\section{Off-diagonal case: $\lambda \in \mathbb{Q}^{+}$}

The only recently achieved solution of the "fractional" local sine-Gordon model [21], involves $N$ quasiparticles with a complex spectrum, whose structure depends on arithmetic properties of the rational sine-Gordon parameter $\lambda$. In the case $\lambda<1$, and introducing the integers $\kappa_{i}$ of the continued fraction decomposition $\lambda=1 /\left[\kappa_{1}+1 /\left\{\kappa_{2}+\ldots\left(+1 / \kappa_{\alpha}\right)\right\}\right]$, the number of modes is $N=1+\sum_{i=1}^{\alpha} \kappa_{i}$. The first quasiparticle is a neutral soliton $A_{\mathrm{s}}$ carrying energy. There are two charged quasiparticles $A_{\mathrm{c}}^{ \pm}$, with charge $\pm q \times q_{\mathrm{s}}$ where $q$ is the denominator of $\lambda$, and a collection of $N-2$ additional neutral particles, carrying only entropy, that are also necessary for the proper description.

Once the correct quasiparticles have been identified, a closed formula for the linear conductance of the Landauer-Büttiker type can be obtained. We here present the general formula [21] valid for the off-diagonal case $\lambda<1$

$$
G(T)=G_{0} \mathcal{A}_{\lambda} \int_{-\infty}^{\infty} d \theta \mathcal{T}_{\mathrm{B}}(\theta)\left[-\partial_{\theta} f_{c}(\theta)\right],
$$

where $\mathcal{A}_{\lambda}$ is a known numerical constant, and $\mathcal{T}_{\mathrm{B}}(\theta)$ is the transmission probability of a charged quasiparticle across the impurity, that is also known. The Fermi factor $f_{c}(\theta)=\frac{1}{1+e^{\epsilon_{c}}}$ is expressed in terms of pseudo energies $\epsilon_{a}(\theta)(a=1, \ldots, N)$ that 

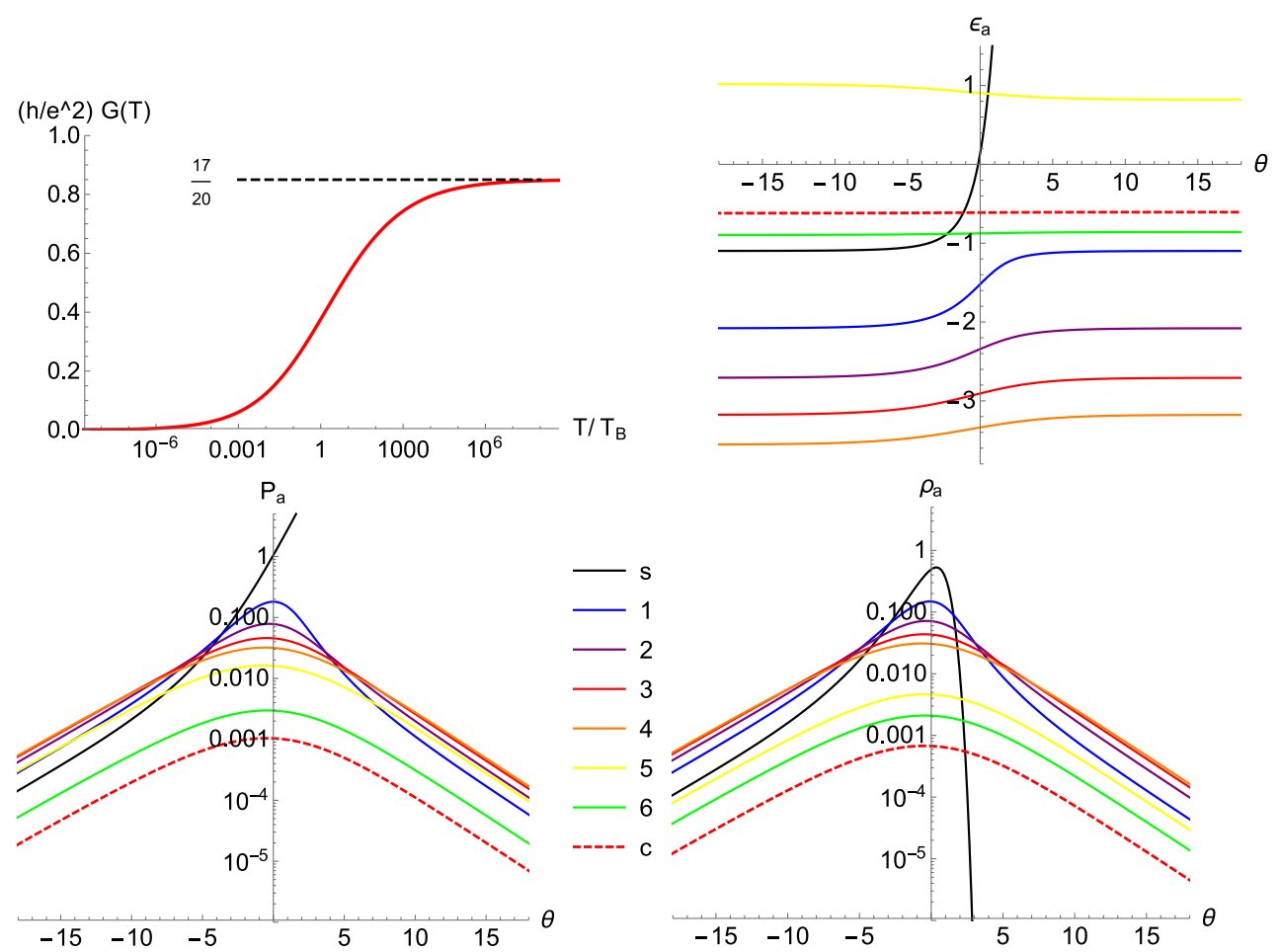

Fig. 2. Top left panel: Exact universal linear conductance $G_{0} G_{K}\left(T / T_{\mathrm{B}}\right)$ of the local sineGordon model for $\lambda=\frac{3}{17}$ or $K=\frac{17}{20}=0.85$, obtained from equations (11) and (12). Top right panel: $\theta$-dependence of the pseudo-energies $\epsilon_{a}$ of the nine quasiparticles that are required to describe the system, shown here in the limit $\frac{V}{T}=0$. The two charged quasiparticle have degenerate spectrum (dashed line). Bottom panel: The corresponding total densities $P_{a}$ of quasiparticles (left) and the densities $\rho_{a}=P_{a} f_{a}$ of occupied quasiparticles (right).

entirely determine the thermodynamical properties of the gas. They are in turn determined by a set of nonlinear coupled integral equations, the thermodynamic Bethe ansatz equations,

$$
\epsilon_{a}(\theta)=\delta_{a, s} e^{\theta}-\frac{1}{2 \pi} \sum_{b=1}^{N} K_{a b} \star \ln \left(1+e^{\mu_{a}-\epsilon_{a}}\right),
$$

where $\star$ denotes a convolution in rapidity space. The kernel $K_{a b}(\theta)$ is known exactly and encodes the effect of the interaction among the quasiparticles. The chemical potential reads $\mu_{a}=q \frac{V}{2 T}\left(\delta_{a, N+1}-\delta_{a, N}\right)$.

We use the case $K=\frac{17}{20}=0.85$ or $\lambda=\frac{3}{17}=1 /[5+1 /(1+1 / 2)]$ involving $1+5+1+2=9$ quasiparticles, to illustrate the procedure for obtaining the conductance. By numerical integration of the thermodynamic Bethe ansatz equation (12) one obtains the pseudo-energies, leading to the conductance $G(T)$ equation (11), see Figure 2. The same procedure but for $\lambda=\frac{1}{3}$ and $\frac{1}{4}$ leads to the dashed lines in Figure 6. 


\section{Spinless fermions on a lattice}

The fermionic many-body model we consider is given by the Hamiltonian

$$
H_{\mathrm{hom}}=-t \sum_{j}\left(c_{j+1}^{\dagger} c_{j}+c_{j}^{\dagger} c_{j+1}\right)+U \sum_{j}\left(n_{j}-1 / 2\right)\left(n_{j+1}-1 / 2\right) .
$$

We used standard second-quantized notation with $c_{j}^{\dagger}$ and $c_{j}$ being creation and annihilation operators on site $j$ respectively and the local density operator $n_{j}=c_{j}^{\dagger} c_{j}$. The nearest-neighbor hopping amplitude is denoted by $t$ and the two-particle interaction by $U$. We shifted the local density in the interacting part by $1 / 2$ such that the Hamiltonian is particle-hole symmetric at half filling $\nu=1 / 2$. The lattice constant is set to $a=1$.

In the thermodynamic limit, if the sums over $j$ in equation (13) run from $-\infty$ to $\infty$ the homogeneous model can be solved by the Bethe ansatz from which the Tomonaga-Luttinger liquid parameter $K(U / t, \nu)$ can be extracted (this also holds for the renormalized velocity $v$, which we, however, are not interested in) [8]. At half filling, which we mainly consider, it is given by

$$
K=\left[\frac{2}{\pi} \arccos \left(-\frac{U}{2 t}\right)\right]^{-1}
$$

The model belongs to the Tomonaga-Luttinger liquid universality class for $-2<$ $U / t<2$. For other fillings $K(U / t, \nu)$ can be computed by numerically solving a set of integral equations and the model is a Tomonaga-Luttinger liquid for all $U / t>-2$ [8]. For later use we report the expansion of $K$ in $U / t$ for arbitrary filling [5]

$$
K=1-\frac{U}{\pi v_{\mathrm{F}}}\left[1-\cos \left(2 k_{\mathrm{F}}\right)\right]+\mathcal{O}\left([U / t]^{2}\right),
$$

with the Fermi velocity $v_{\mathrm{F}}=2 t \sin k_{\mathrm{F}}$ and $k_{\mathrm{F}}=\nu \pi$.

The formalism we use below to compute the temperature dependence of the linear conductance of the model equation (13) complemented by a local impurity, employs a transport geometry in which the interacting wire is connected to two semi-infinite noninteracting leads. We restrict the interaction to $N-1$ bonds between the sites $j \in[1, N]$ and in the second sum in equation (13) the site index thus runs from $j=1$ to $j=N-1$. However, the lattice site in the first sum runs from $-\infty$ to $\infty$. The sites $j \leq 0$ form a left lead and the sites $j \geq N+1$ a right one. Due to the abrupt change of the two-particle interaction at the two contacts at sites $j=1$ and $j=N$ the $T=0$ conductance of the setup does not take the unitary value. The inhomogeneity of the two-particle term leads to a single-particle backscattering which masks the effect of the single impurity to be introduced into the interacting part of the wire. We therefore smoothly turn off the interaction over $N_{\mathrm{c}} \ll N$ lattice sites when approaching the contacts at $j=1$ and $j=N$ from the center on the interacting chain. As discussed in reference [42] the details of the envelope function do not matter as long as it is sufficiently smooth. This way the $T=0$ conductance in the absence of an impurity can be tuned arbitrarily close to the unitary limit. In the numerical results shown below, me made sure that the relative deviation from the unitary conductance is less than $10^{-4}$. Note that for this setup with "adiabatically connected" noninteracting leads the unitary conductance is given by $G_{0}=e^{2} / h$, instead of $K e^{2} / h$ as obtained for the model of Section 2 in which the interaction is not restricted to a subsystem [42-44]. One can expect that this difference does not 
affect the universal properties after adding a local impurity. Below we will confirm this by directly comparing the conductance and the $\beta$-function of the present model with the one of the local sine-Gordon model.

The system is complemented by a hopping impurity on the bond from site $N / 2$ to site $N / 2+1$ ( $N$ even) with the Hamiltonian

$$
H_{\mathrm{hop}}=t^{\prime}\left(c_{N / 2+1}^{\dagger} c_{N / 2}+c_{N / 2}^{\dagger} c_{N / 2+1}\right)
$$

or a site impurity with $H_{\text {site }}=V n_{(N+1) / 2}, N$ odd. In the first case the total hopping across the central bond is $t-t^{\prime}$. A $t^{\prime} \neq 0$ or $V \neq 0$ leads to a nonvanishing impurity backscattering. For $U=0$ the transmission amplitude and thus $G(T)$ for both types of impurities can be computed exactly using single-particle scattering theory (see, e.g. Ref. [45]).

To compute $G(T)$ of the lattice model for $U \neq 0$ we use Matsubara Green functions and the functional RG approach [46]. The technical details of the application of this method to study transport properties of lattice models of inhomogeneous TomonagaLuttinger liquids are given in references [47-49]. We here only present the basic idea. The relevant steps are the following:

1. Express the partition function as a coherent state functional integral.

2. Integrate out the noninteracting leads by projection. They are incorporated exactly as lead self-energies in the propagator of the interacting part.

3. Replace the reservoir-dressed noninteracting propagator of the system by one decorated by a cutoff $\Lambda$. For the initial value $\Lambda_{i}$, the free propagation must vanish; for the final one $\Lambda_{\mathrm{f}}$, the original propagation must be restored. One often uses a cutoff in the Matsubara frequency. When $\Lambda$ is sent from $\infty$ to 0 this incorporates the RG idea of a successive treatment of energy scales.

4. Differentiate the generating functional of one-particle irreducible vertex functions with respect to $\Lambda$.

5. Expand both sides of the functional differential equation with respect to the one-particle irreducible vertex functions. This leads to an infinite hierarchy of coupled differential equations for the vertex functions.

The hierarchy of coupled flow equations presents an exact reformulation of the quantum many-body problem. Integrating it from $\Lambda_{\mathrm{i}}$ to $\Lambda_{\mathrm{f}}$ leads to exact expressions for the vertex functions from which observables such as the conductance can be computed. In practice, truncations of the hierarchy are required, resulting in a closed finite set of equations. The integration of these leads to approximate expressions for the vertices and, thus, for observables.

Truncating the infinite hierarchy of equations we neglect the flow of three- and higher particle vertex functions and replace the two-particle vertex by a static one of nearest-neighbor-type. The resulting flow equations and the expression of $G(T)$ in terms of the vertex functions can be found in references $[47,48]$. In this truncation the self-energy (single-particle vertex function) is independent of the Matsubara frequency and can be interpreted as an effective single-particle potential which is generated during the RG flow by the interplay of the bare impurity and the two-particle interaction. At the end of the flow (for $\Lambda_{\mathrm{f}}$ ) a single-particle scattering problem in the presence of the effective potential has to be solved. This provides a comparatively simple picture of a many-particle correlation effect. Integrating the coupled flow equations for the effective interaction and the self-energy in the limits $t^{\prime} \rightarrow 0$ or $V \rightarrow 0$ (weak impurity) and $t^{\prime} \rightarrow t$ or $V \rightarrow \infty$ (weak link) it was shown analytically 

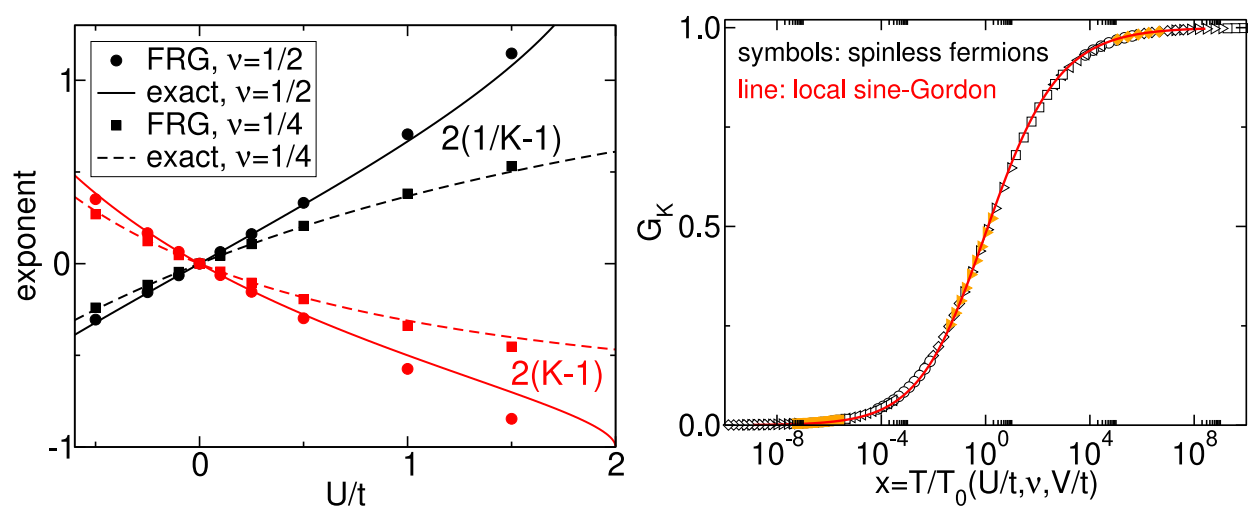

Fig. 3. Left panel: Strong (black) and weak (red) impurity exponents as a function of $U / t$ for two different fillings $\nu=1 / 2$ and $1 / 4$. Right panel: One-parameter scaling plot of the conductance. Open symbols represent results obtained for $U / t=0.5, \nu=1 / 2$, and different $T$ and amplitudes of the site impurity $V$, while filled symbols were calculated for $U / t=0.851, \nu=1 / 4$. Both pairs of $U / t$ and $\nu$ lead to the same $K=0.85$. The solid lines shows the universal conductance curve of the local sine-Gordon model for $K=0.85$ (see the upper left panel of Fig. 2).

that the approximate functional RG reproduces the power-law scaling underlying equations (2) and (3) [50]. In the effective single-particle picture the power laws follow from scattering off an effective potential which during the RG flow develops a long-ranged oscillatory part $[47,48]$ (see also Ref. [23]) out of the purely local bare impurities (hopping or site). Further away from the bare impurity the long-range part leading to the power-law scaling of $G(T)$ is cut off at a scale $\sim 1 / T$.

In the left panel of Figure 3 we show the exponents $2(K-1)$ and $2(1 / K-1)$ as functions of $U / t$ for two different fillings, obtained by inserting the Bethe ansatz result for $K$ ("exact") as well as by numerically computing $G(T)$ from the functional RG and extracting the exponent ("FRG"). For sufficiently small $|U / t|$ the agreement is excellent, and the deviations from the exact results are small up to moderate values of the interaction. In fact, the extracted exponents agree to leading order in $U / t$ to the result obtained when inserting the expansion equation (15) for $K$.

The right panel of Figure 3 shows a one-parameter scaling plot of the conductance in the presence of a site impurity as a function of $x=T / T_{0}(U / t, \nu, V / t)$ with a nonuniversal scale $T_{0}(U / t, \nu, V / t)[14,17-19,48,51]$. Open symbols were computed for $\nu=1 / 2$ and $U / t=0.5$, while filled ones were computed for $\nu=1 / 4$ and $U / t=0.851$. For both parameter sets one obtains $K \approx 0.85$ (within the approximated functional RG). For appropriately chosen $T_{0}$ (determined "by hand") the $G(T) / G_{0}$ curves for different $V, \nu$, and $U$, but fixed $K$, collapse onto a $K$-dependent, dimensionless scaling function $G_{K}(x)$. Note that the data extend over roughly 20 orders of magnitude in temperature. In accordance with equations (2) and (3) $G_{K}(x)$ exhibits the limiting behavior $G_{K}(x) \propto 1-x^{2(K-1)}$ for $x \rightarrow \infty$ and $G_{K}(x) \propto x^{2(1 / K-1)}$ for $x \rightarrow 0$. We note that to prevent deviations from scaling $T$ must be taken much smaller than the band width $4 t$ - the high-energy cutoff of the present model - and much larger than a scale of order $1 / N$ which cuts off the universal behavior at the lower end. In the figure $N=10^{4}$ was considered such that, for fixed parameters, several orders of magnitude in $T$ can be used for the scaling plot. We note that in addition data for a hopping impurity can be collapsed on the curve (not shown). For fixed $K$ this provides a "numerical proof" of universality, that is independence on the details of the impurity and its amplitude, the interaction strength $U / t$ as well as the filling 
$\nu$, within the lattice model. For the local sine-Gordon model this type of "internal" universality can be shown analytically; see Section 2 .

In addition to the functional $\mathrm{RG}$ data for the spinless fermion model (symbols) the right panel of Figure 3 shows the universal linear conductance (divided by $G_{0}=$ $K e^{2} / h$ ) of the local sine-Gordon model (red line; the same data as in the upper left panel of Fig. 2). The agreement is excellent. It is plausible to assume that the small deviations result out of the approximate nature of our functional RG approach to the spinless fermion model which is only controlled for small to intermediate $|U / t|$. Decreasing $|U / t|$, i.e. bringing $K$ closer to one, further reduces the deviation. This indicates that the low-energy physics of both models, with $T \ll W$ for the local sine-Gordon model and $v_{\mathrm{F}} / N \ll T \ll 4 t$ for the lattice model of spinless fermions with an impurity, is dominated by the same quantum critical point. The excellent agreement "proves" universality across the models. We here compare the $G_{K}(x)$ of the two models, and not the $\beta$-function, as it has a direct physical meaning (the conductance).

After introducing the experimental quantum circuit as our third system to realize the quantum critical point in the next section, we will show that the $\beta$-function, in which the nonuniversal scale is eliminated, is the same in all cases and, therefore, universality holds between all three systems. This will also confirm our expectation, that $\beta_{K}$ is a function of $g$ only, see Section 1.4.

\section{A tunable quantum circuit: experiments}

A quantum circuit composed of a short spin-polarized electronic channel in series with a resistance (see schematic in Fig. 4) appears markedly different from a system of interacting electrons confined to 1d. Nevertheless, at low temperatures, this circuit is predicted to be mathematically described by the same local sine-Gordon model [12] as a 1d metal of spinless fermions including a single impurity [5]. Such a connection illustrates the remarkable universal character of the Tomonaga-Luttinger liquid concept. It originates from the similar continua of bosons describing, on the one hand, $1 \mathrm{~d}$ fermions with short-range interactions $[5,8]$ and, on the other hand, a linear resistance $R$ in a quantum circuit [52]. As a result, the two different systems can be mapped onto the same Tomonaga-Luttinger Hamiltonian with interaction parameter $K=1 /\left(1+R e^{2} / h\right)[12]$. In practice, the quantum circuit implementation $[20,26,53,54]$ constitutes an experimental test-bed that stands out in that it allows for direct, quantitative and parameter-free investigations of the universal Tomonaga-

Luttinger liquid physics; see our discussion in Section 1.2 for other realization of Tomonaga-Luttinger liquids.

Quantum circuits can be engineered or adjusted in-situ to cover arbitrary strengths of repulsive interactions $0<K<1$, through the tuning and separate characterization of $R[20,26,53,54]$. For the data discussed in this minireview, we used a robust and precise approach that consists in implementing a linear resistance $R=h / n e^{2}$ from $n \in \mathbb{N}$ integer quantum Hall edge channels in parallel $[20,54]$. Correspondingly, the interaction parameter can take the values $K=n /(n+1)$, with here $n \in\{1,2,3,4\}$. Note that lower values of $K$ can be achieved through larger resistances obtained with narrow metallic stripes (instead of ballistic channels) [53]. On a practical side, such stripes should be short enough so that any distributed capacitance remains negligible with respect to $R$ up to the relevant frequency scale $k_{\mathrm{B}} T / h$, with $k_{\mathrm{B}}$ the Boltzmann constant.

The impurity is realized by a quantum point contact formed in a $\mathrm{Ga}(\mathrm{Al}) \mathrm{As}$ two dimensional electron gas, see Figure 1 of reference [20] for an electron micrograph of the device schematically represented in Figure 4. The spin polarization is achieved 


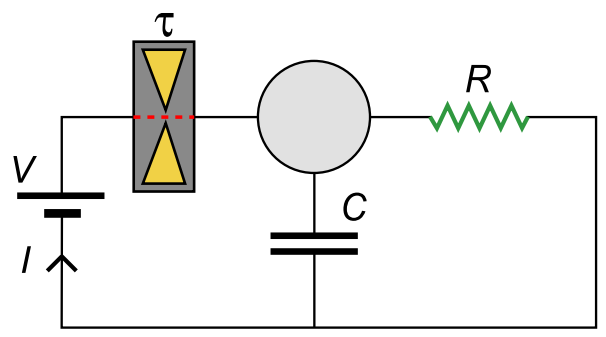

Fig. 4. Schematic representation of a quantum circuit described at low temperature $\left(k_{\mathrm{B}} T \ll\right.$ $h / R C)$ by the same Hamiltonian as the local sine-Gordon model and the model $1 \mathrm{~d}$ spinless fermions with an impurity. In practice, a series resistance $R=h / n e^{2}$ is implemented from the parallel combination of $n$ quantum Hall edge channels.

by immersing the device in a large perpendicular magnetic field of $2.7 \mathrm{~T}$ (corresponding to the regime of the integer quantum effect at filling factor 3). The impurity quantum point contact is tuned in-situ, by field effect, to partially transmit a single electronic channel (the outer quantum Hall edge channel). It is characterized by the unrenormalized transmission probability $\tau$ of electrons across the channel, which is experimentally obtained from the quantum point contact differential conductance $G_{\mathrm{QPC}}=\tau e^{2} / h$ measured at large enough dc voltage bias to suppress the Tomonaga-Luttinger (dynamical Coulomb blockade) conductance reduction.

The interconnection between the quantum point contact and series resistance $R=h / n e^{2}$ involves a micron-scale metallic island playing the role of a floating reservoir merging all quantum Hall channels, which would otherwise separately propagate along opposite sample edges. Note that the metal is thermally diffused into the GaAsGaAlAs heterojunction, in order to make a good electrical contact with the two dimensional electron gas located approximately $100 \mathrm{~nm}$ below the surface. The geometrical self-capacitance of the island $C \simeq 3.1 \mathrm{fF}$ determines the relevant high-energy cutoff, with universal Tomonaga-Luttinger behavior emerging at $k_{\mathrm{B}} T \ll h /(R C)$. The knowledge of the important cutoff parameter $C$ is experimentally obtained from Coulomb diamond measurements of the conductance across the same island, but with all connected quantum point contacts tuned to weak tunnel couplings [20].

This circuit can be described by the following Hamiltonian:

$$
H=H_{0}+H_{\mathrm{I}}+H_{\mathrm{C}}+H_{\mathrm{env}} .
$$

Here $H_{0}$ represents a ballistic conduction channel

$$
H_{0}=i \hbar v_{\mathrm{F}} \int d x\left(\psi_{+}^{+} \partial_{x} \psi_{+}-\psi_{-}^{+} \partial_{x} \psi_{-}\right)
$$

where $\psi_{+(-)}$is the annihilation operator for the electrons moving toward (away from) the island and $v_{\mathrm{F}}$ is the Fermi velocity. $H_{\mathrm{I}}$ models the backscattering at the QPC located at $x=0, H_{\mathrm{I}}=\hbar v_{\mathrm{F}} r\left[\psi_{+}^{+}(0) \psi_{-}(0)+\psi_{-}^{+}(0) \psi_{+}(0)\right]$, with $|r|^{2} \simeq 1-\tau$ for a near ballistic QPC. $H_{\mathrm{C}}$ is the coupling between electrons in the channel and the electromagnetic RC environment, $H_{\mathrm{C}}=-\hat{Q}\left(V-\partial_{t} \hat{\Phi}\right)$ with $\hat{Q}$ the total charge transferred across the QPC and $\hat{\Phi}$ a bosonic operator corresponding to the time integral of the voltage across the RC impedance. The dissipative $\mathrm{RC}$ environment $H_{\text {env }}$ is modeled by an infinite set of quantum LC resonators as detailed in [52]. The mapping of such a circuit on the spinless Tomonaga-Luttinger Hamiltonian including an impurity was first demonstrated in [12]. 

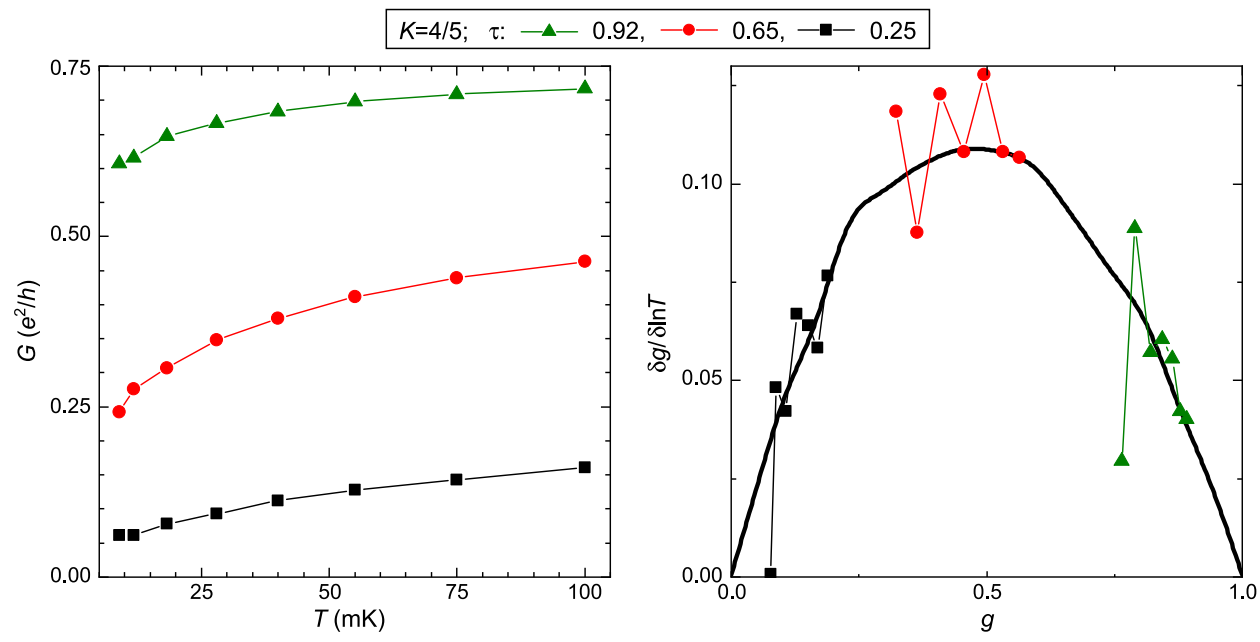

Fig. 5. Experimental procedure illustrated at $K=4 / 5\left(R=h / 4 e^{2}\right)$ for three settings of the impurity backscattering $\tau$. Left panel: Symbols represent measurements of the conductance across the device plotted versus temperature. Right panel: Discrete differentiation $\delta g / \delta \ln T$ plotted versus $g=G /\left(K e^{2} / h\right)$. Symbols connected by lines are obtained from the conductance data of corresponding color in the left panel. The thick continuous lines represent a low-pass Fourier averaging of the full data set $(\sim 200$ values of $\tau)$.

The prominent observable for electronic systems is the electrical conductance. In the present context of a Tomonaga-Luttinger liquid with an impurity, a crossover from a conductor toward an insulator is predicted to develop as the temperature is reduced, except in the absence of interactions $K=1(R=0)$ or in the absence of any impurity $(\tau=1)[13,14]$. According to the (inhomogeneous) Tomonaga-Luttinger liquid theory, and as shown explicitly in Section 3, the conductance $G$ along this crossover follows a universal scaling law that only depends on the interaction parameter $K$ [14].

Experimentally, for all implemented interaction parameter $K=1 /\left(1+R e^{2} / h\right) \in$ $\{1 / 2,2 / 3,3 / 4,4 / 5\}$, the impurity backscattering strength is spanned over the full range $\tau \in[0,1]$. At each given setting of $\tau$, we measure the conductance $G$ of the device (quantum point contact and series resistance) at several values of the temperature, as illustrated for $K=4 / 5\left(R=h / 4 e^{2}\right)$ in the left panel of Figure 5 with $\tau \simeq 0.25$ (black), 0.65 (red) and 0.92 (green). Then, using data points up to temperatures of at most $h /\left(25 k_{\mathrm{B}} R C\right)$ (in order to ascertain a universal low energy behavior), we perform discrete differentiations $\delta g / \delta \ln T$ for each given device setting of $\tau$. The result is plotted as a function of $g$ in the right panel of Figure 5, as symbols connected by lines corresponding to the conductance data of the same color in the left panel. This procedure is repeated for many settings of $\tau$, approximately 200 values for each $K$. As shown in Figure 2(a) of reference [20], all data points for a given $K$ (series resistance) pile up on the same curve independently of the device's tuning of $\tau$, which directly demonstrates an underlying universal scaling behavior. The corresponding experimental renormalization flow $\beta$-function $\beta_{K}(g)$, shown as thick continuous lines in the right panel of Figure 5 for $K=4 / 5$ and in Figure 6 for $K=3 / 4$ and 4/5, were obtained by a low-pass Fourier averaging of the individual data points (for $K=1 / 2$ and 2/3 see Figure 2(a) of reference [20] including a comparison with exact solutions of the local sine-Gordon model). The quantitative agreement reached between the experimentally derived $\beta_{K}(g)$ using a quantum circuit implementation (colored continuous lines), the exact theoretical solutions of the local sine-Gordon model (black dashed lines), and the functional RG results for a lattice model of spinless fermions 


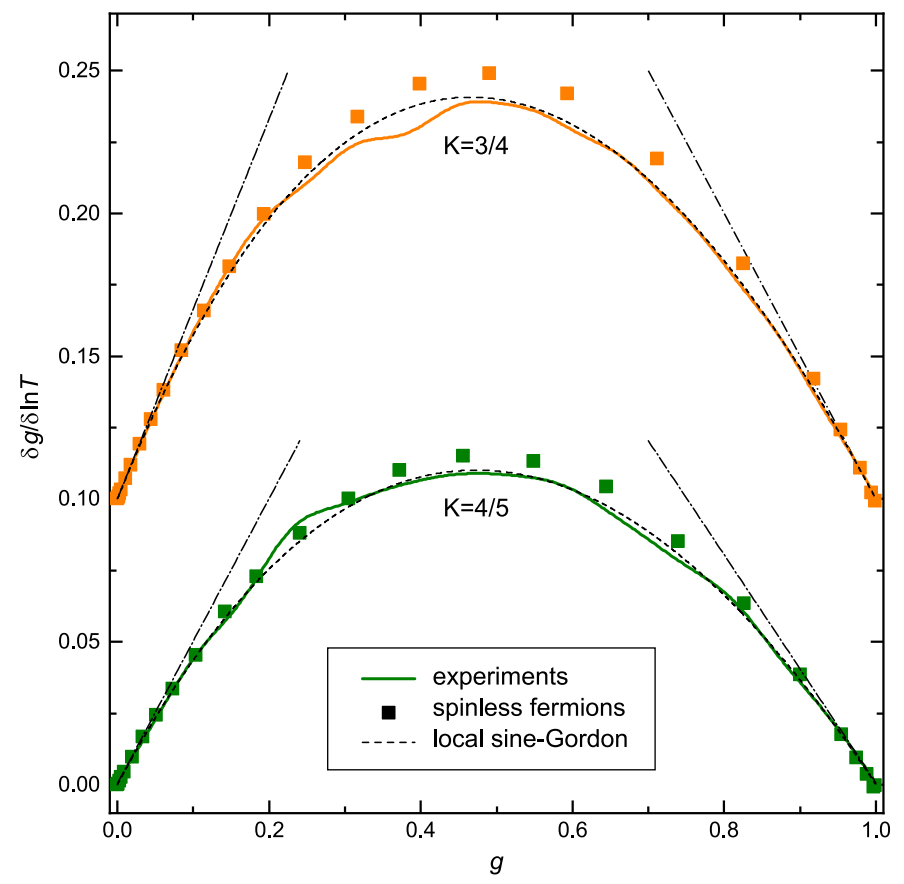

Fig. 6. Comparison of the renormalization flow $\beta$-functions at $K=3 / 4$ (shifted vertically by 0.1 ) and $4 / 5$ obtained experimentally by measuring a quantum circuit (colored continuous lines), numerically by computing the conductance employing an approximate functional RG approach to a model of interacting spinless fermions with one impurity (symbols), and analytically by solving exactly the local sine-Gordon model (blacked dashed lines). The asymptotic behavior equations (5) and (6) for $1-g \ll 1$ and $g \ll 1$, respectively, is indicated by the dashed-dotted lines.

with one impurity (symbols) is displayed in Figure 6. We emphasize that the comparison is free of any fitting parameters. As expected (see Sect. 1.4) the unique $\beta$-function depends on the temperature only via the dimensionless conductance $g$. The parameter free collapse of all data sets provides a convincing example of the power of emergent universality originating from an underlying quantum critical point. This universality provides a bridge between different fields of physics, here between field theory, quantum many-body theory of correlated Fermi systems, and experimental circuit quantum electrodynamics.

\section{Summary and outlook}

There exist at least three levels from which the results presented in our minireview can be summarized.

The highest one is the perspective of emergent universality in complex systems resulting out of a common underlying (quantum) critical point. It bridges different fields of physics. Our theoretical results obtained for the field theoretical local sineGordon model and the condensed matter model of spinless lattice fermions with nearest-neighbor hopping, nearest-neighbor interaction and a local impurity, as well as the experiments on highly tunable quantum electrodynamical circuits provide a convincing example of the power of this concept. The parameter free collapse of the low-energy $\beta$-function of the conductance for different $K$, as shown in Figure 6 , is 
of exceptional high quality. It explicitly shows that the underlying quantum critical point is the same for all three systems.

The second level is the one of the physics of a local impurity in a $1 \mathrm{~d}$ correlated Fermi systems. Considering the two different models and the experimental emulation we were able to shed light on the emergence of the metal-to-insulator transition. Even if the bulk system is metallic the interplay of the single impurity and a repulsive twoparticle interaction will drive it into an insulating state. The quantum critical nature of the transition leads to the universality within a given system. For fixed $K$, the microscopic details and parameters enter the conductance only via a nonuniversal scale $T_{0}$.

The third level concerns the experimental verification of the (inhomogeneous) Tomonaga-Luttinger liquid concept. Our discussion shows that to convincingly demonstrate this type of physics requires experimental control, tunability, and the access to the variable in which power-law scaling is to be shown (the rescaled temperature $T / T_{0}$ with a temperature scale $T_{0}$ encapsulating microscopic details) over several orders of magnitude. In this respect the emulation by quantum circuits is clearly superior to the attempts to directly realize (quasi-) 1d fermionic systems in semi-conductor-based heterostructures, self-organized atom chains on surfaces, and unidirectional long molecules (e.g. metallic carbon nanotubes). We are not aware of any experiments on such systems which show Tomonaga-Luttinger liquid behavior in a way which is equally convincing as the emulations discussed here (see also Ref. [26]). In fact, many of the interpretations of experimental data on (quasi-) 1d fermionic systems in the light of Tomonaga-Luttinger liquid theory have been questioned.

As a next step it would be very interesting to emulate other models of inhomogeneous Tomonaga-Luttinger liquids. Even introducing a second localized impurity is expected to lead to new effects associated to resonant transport (see Refs. $[24,48]$ and references therein). In this case no exact solution for any model properly describing this situation is available. In fact, different approximate approaches lead to conflicting results on the emergence of a regime of energies with a novel scaling exponent. The experimental emulation of this setup could provide a very useful contribution to settle this issue. Novel fixed points with unique scaling exponents have also been predicted for Y-junctions of three Tomonaga-Luttinger liquids (see Ref. [24] and references therein). Experimentally realizing those would also be of great interest. Furthermore, experimentally emulating correlated 1d fermions in (more) complex nonequilibrium situations, such as periodically driven systems, could help to deepen our understanding of the interplay of two-particle interactions and nonequilibrium. Currently, the number of tools to investigate such systems theoretically in a controlled way is very limited.

Open access funding provided by Projekt DEAL.

Open Access This is an open access article distributed under the terms of the Creative Commons Attribution License (http://creativecommons.org/licenses/by/4.0), which permits unrestricted use, distribution, and reproduction in any medium, provided the original work is properly cited.

\section{References}

1. A.L. Fetter, J.D. Walecka, Quantum Theory of Many-Particle Systems (McGraw-Hill, New York, 1971)

2. A. Luther, I Peschel, Phys. Rev. B 9, 2911 (1974)

3. D.C. Mattis, J. Math. Phys. 15, 609 (1974)

4. J. Sólyom, Adv. Phys. 28, 201 (1979) 
5. T. Giamarchi, Quantum Physics in One Dimension (Oxford University Press, New York, 2003)

6. J. von Delft, H. Schoeller, Ann. Phys. 7, 225 (1998)

7. K. Schönhammer in Interacting Electrons in Low Dimensions, edited by D. Baeriswyl (Kluwer Academic Publishers, Dordrecht, 2005)

8. F.D.M. Haldane, Phys. Rev. Lett. 45, 1358 (1980)

9. M. Grioni, S. Pons, E. Frantzeskakis, J. Phys.: Condens. Matter 21, 023201 (2009)

10. V.V. Deshpande, M. Bockrath, L.I. Glazman, A. Yacoby, Nature 464, 209 (2010)

11. T. Giamarchi, Int. J. Mod. Phys. B 26, 1244004 (2012)

12. I. Safi, H. Saleur, Phys. Rev. Lett. 93, 126602 (2004)

13. W. Apel, T.M. Rice, Phys. Rev. B 26, 7063 (1982)

14. C.L. Kane, M.P.A. Fisher, Phys. Rev. B 46, 15233 (1992)

15. S. Chakravarty, Phys. Rev. Lett. 49, 681 (1982)

16. A. Schmid, Phys. Rev. Lett. 51, (1983) 1506

17. K. Moon, H. Yi, C.L. Kane, S.M. Girvin, M.P.A. Fisher, Phys. Rev. Lett. 71, 4381 (1993)

18. K. Leung, R. Egger, C.H. Mak, Phys. Rev. Lett. 75, 3344 (1995)

19. P. Fendley, A.W.W. Ludwig, H. Saleur, Phys. Rev. B 52, 8934 (1995)

20. A. Anthore, Z. Iftikhar, E. Boulat, F.D. Parmentier, A. Cavanna, A. Ouerghi, U. Gennser, F. Pierre, Phys. Rev. X 8, 031075 (2018)

21. E. Boulat, arXiv:1912.03872

22. S. Eggert, I. Affleck, Phys. Rev. B 46, 10866 (1992)

23. D. Yue, L.I. Glazman, K.A. Matveev, Phys. Rev. B 49, 1966 (1994)

24. V. Meden, S. Andergassen, T. Enss, H. Schoeller, K. Schönhammer, New J. Phys. 10, $045012(2008)$

25. S. Sachdev, Quantum Phase Transitions (Cambridge University Press, Cambridge, 2011)

26. H.T. Mebrahtu, I.V. Borzenets, D.E. Liu, H. Zheng, Y.V. Bomze, A.I. Smirnov, H.U. Baranger, G. Finkelstein, Nature 488, 61 (2012)

27. S. Ghoshal, A. Zamolodchikov, Int. J. Mod. Phys. A 09, 3841 (1994)

28. P. Fendley, H. Saleur, Nucl. Phys. B 428, 681 (1994)

29. P. Fendley, H. Saleur, N. Warner, Nucl. Phys. B 430, 577 (1994)

30. A.B. Zamolodchikov, A.B. Zamolodchikov, Ann. Phys. 120, 253 (1979)

31. L.D. Faddeev, Sov. Sci. Rev. C 1, 107 (1980)

32. A.B. Zamolodchikov, Nucl. Phys. B 342, 695 (1990)

33. A. Zamolodchikov, Phys. Lett. B 253, 391 (1991)

34. M. Takahashi, M. Susuki, Prog. Theor. Phys. 48, 2187 (1972)

35. M. Takahashi, Thermodynamics of one-dimensional solvable models (Cambridge University Press, Cambridge, 2005)

36. V. E. Korepin, G. Izergin, N.M. Bogoliubov, Quantum Inverse Scattering Method and Correlation Functions (Cambridge University Press, Cambridge, 1993)

37. L.D. Faddeev, in Symétries Quantiques/Quantum Symmetries: Les Houches, Session LXIV, edited by A. Connes, K. Gawedzki, J. Zinn-Justin (North-Holland Publishing, Amsterdam, 1998)

38. M. Fowler, X. Zotos, Phys. Rev B 25, 5806 (1982)

39. G. Chung, Y.C. Chang, Phys. Rev. Lett. 50, 791 (1983)

40. P. Fendley, K. Intriligator, Nucl. Phys. B 372, 533 (1992)

41. R. Tateo, Int. J. Mod. Phys. A 10, 1357 (1995)

42. K. Janzen, V. Meden, K. Schönhammer, Phys. Rev. B 74, 085301 (2006)

43. I. Safi, H.J. Schulz, Phys. Rev. B 52, R17040 (1995)

44. D.L. Maslov, M. Stone, Phys. Rev. B 52, R5539 (1995)

45. V. Meden, P. Schmitteckert, N. Shannon, Phys. Rev. B 57, 8878 (1998)

46. W. Metzner, M. Salmhofer, C. Honerkamp, V. Meden, K. Schöhammer, Rev. Mod. Phys. 84, 299 (2012)

47. V. Meden, T. Enss, S. Andergassen, W. Metzner, K. Schönhammer, Phys. Rev. B 71, $041302(2004)$ 
48. T. Enss, V. Meden, S. Andergassen, X. Barnabé-Thériault, W. Metzner, K. Schönhammer, Phys. Rev. B 71, 155401 (2005)

49. S. Andergassen, T. Enss, V. Meden, W. Metzner, U. Schollwöck, K. Schönhammer, Phys. Rev. B 73, 045125 (2006)

50. V. Meden, W. Metzner, U. Schollwöck, K. Schönhammer, J. Low Temp. Phys. 126, $1147(2002)$

51. V. Meden, S. Andergassen, W. Metzner, U. Schollwöck, K. Schönhammer, Europhys. Lett. 64, 769 (2003)

52. H. Grabert, M.H. Devoret (eds.), Single charge tunneling (Plenum Press, New York, 1992)

53. F.D. Parmentier, A. Anthore, S. Jezouin, H. le Sueur, U. Gennser, A. Cavanna, D. Mailly, F. Pierre, Nat. Phys. 7, 935 (2011)

54. S. Jezouin, M. Albert, F. D. Parmentier, A. Anthore, U. Gennser, A. Cavanna, I. Safi, F. Pierre, Nat. Commun. 4, 1802 (2013) 